\title{
The apparent length of rotating arcs under conditions of dark adaptation*
}

\author{
A. J. MARSHALL \\ University of Western Australia, Nedlands, Western Australia 6009 \\ and \\ GORDON STANLEY \\ University of Melbourne, Parkville, Victoria 3052, Australia
}

\begin{abstract}
The apparent contraction of a rotating light arc occurred during the first $20 \mathrm{~min}$, but not after $25 \mathrm{~min}$, of dark adaptation. Estimates of length obtained after $25 \mathrm{~min}$ were affected by the level of luminance of the arc but not by its speed of rotation, by dark gaps in the arc, or by instructions to estimate its length in terms of a brighter region. There was no tendency for a rotating dark arc to appear shorter at any stage of adaptation.
\end{abstract}

Ansbacher (1964) reported that a narrow slot in an opaque disk, seen as a light arc line when lit from behind, appeared to shrink to a fraction of its actual length when rotated in a frontoparallel plane. When fixation was directed toward the center of the disk, the apparent contraction of the arc increased with rotation speeds up to $80 \mathrm{rpm}$. A similar effect was obtained when viewing by reflected light a white arc line set on a rotating black disk, but no apparent shrinkage occurred with a black are line on a rotating white disk (Marshall \& Stanley, 1964). No contraction occurred when viewing by transmitted light an opaque arc line set on a rotating translucent disk (Stanley, 1966).

Graham (1963) considered that the short leading edge of a narrow rectangle of light in motion would give rise to local contrast effects with possibly an increase in brightness. The trailing edge would be less sharpened by contrast, and the end part of the line would be somewhat dimmer from inhibitory effects. Two studies (Stanley, 1967a; Stanley \& Jackson, 1969) provide some evidence for the proposition that the leading edge is critical for a contraction effect when a narrow light arc is rotated. In another study (Stanley, 1967b), an increase of brightness with increasing speed over a range for which increasing contraction occurs was found. Stanley (1967b) proposed that the apparent contraction of rotating light arcs might be due to brightness integration and a funneling of excitation into a smaller spatial region (Békésy, 1967). The brighter region produced by onset of stimulation due to movement of the leading edge is assumed to inhibit dimmer regions so that the impression of length of the moving line corresponds more with the extent of the brighter region.

\footnotetext{
*These experiments were conducted at the University of Western Australia. They were supported by ARGC Grant A67/16441 to the second author. Requests for reprints should be addressed to either A. J. Marshall, Department of Psychology, University of Western Australia, Nedlands, Western Australia 6009 , or Gordon Stanley, Department of Psychology, University of Melbourne, Parkville, Victoria 3052, Australia.
}

A decrease in lateral inhibition or an increase of areal summation has been used to account for the changes both in light sensitivity and in the light thresholds for given levels of contour acuity during the course of dark adaptation (Barlow, 1964; Lythgoe, 1940). Békésy (1968) reported that the Mach band effect does not occur under conditions of dark adaptation owing to an absence of inhibitory spatial interaction. This raises the question of whether the contraction of the apparent length of moving light arcs found with daylight vision also occurs under conditions of dark adaptation.

Two experiments were carried out. In the first, estimates of the length of a light arc moving on a dark surround and of a dark arc on a light surround were obtained as the arcs themselves became visible at different luminance levels during the course of dark adaptation. Length estimates of the rotating arcs were also obtained at various levels of luminance after $25 \mathrm{~min}$ of dark adaptation. The second experiment checked on the possible effect of instructions to judge the apparent length of a rotating light arc in terms of any brighter part of the display after the $S$ had been completely, or almost completely, dark-adapted. The effects of the length of the light arc, its level of luminance, the presence or absence of gaps, and its speed of rotation were explored, also after $25 \mathrm{~min}$ of dark adaptation.

\section{EXPERIMENT I LIGHT AND DARK ARCS}

\section{Method}

\section{Subjects}

Six Ss, aged 20-25, who had no marked visual defects and no previous experience in the experimental situation, attended individually at two sessions of approximately $1 \frac{1}{2} \mathrm{~h}$ each.

\section{Stimuli}

Two circular disks, $500 \mathrm{~mm}$ in diam and one wooden and one 
of translucent perspex. were used to present the stimuli. The wooden disk was painted black and had two slots $120 \mathrm{~mm}$ long and $8 \mathrm{~mm}$ wide. One slot, an arc of a circle of radius $180 \mathrm{~mm}$, and the other, an arc of radius $210 \mathrm{~mm}$, were at these radial distances from the center of the disk. Various lengths of arc could be obtained by shields that covered all but one light arc of the required length and radial position. For the dark arcs on light surrounds, black metal arcs of the required length could be clipped into the perspex disk at radial distances of 180 and $210 \mathrm{~mm}$. A small red reflector. $5 \mathrm{~mm}$ in diam, was set at the center of each disk to serve as a fixation point.

\section{Apparatus}

The apparatus was housed in a dark room. Its main features were: (a) an enclosed optical bench, with a traveling light, a 6-V $32-W$ headlamp bulb, run at a constant voltage from storage batteries; (b) an optical system with shutter to project an even circular patch of light onto the test surface covering the stimulus area; (c) a mounting with a friction drive to rotate either of the stimulus disks at $60 \mathrm{rpm}$ in a frontoparallel plane; (d) a cubicle on the further side of stimulus disk, with a white bowl for light adapting the $S$ and a chinrest giving a viewing distance of $800 \mathrm{~mm}$ from the stimulus disk. A light, adjusted by a rheostat, was projected from behind the S's head onto the reflector at the center of each stimulus disk. The levels of relative luminance emittance from the test surface, for different positions of the light along the optical bench, were determined by means of a photomultiplier; the luminance of the bowl used for light adaptation was approximately $1,700 \mathrm{~cd} / \mathrm{m}^{2}$ as measured by an SEI photometer.

\section{Procedure}

At his first session, the $S$ carried out two dark-adaptation trials of $30-40$ min each, during which he was required to judge the orientation of a contour acuity test object. The adaptation curves obtained from all Ss were similar and showed no abnormalities. In his next session, each $\mathrm{S}$ was allocated at random to either of two groups. One group was instructed to detect a dark arc line rotating on a light background and to make estimates of its length in inches or fractions of an inch, at different times during the course of dark adaptation. Each $S$ was given a few practice trials with stationary arc lines varying in length from 30 to $180 \mathrm{~mm}$. Then he was light-adapted for $3 \mathrm{~min}$, after which the rotating arc was presented for $2 \mathrm{sec}$ (a buzzer sounding after $1 \mathrm{sec}$ ) at intervals of approximately $15 \mathrm{sec}$ throughout the period of dark adaptation. The $S$ was told to fixate the center spot and to make an estimate of arc length as soon as the buzzer sounded. The first testing luminance was $2.5 \mathrm{~cd} / \mathrm{m}^{2}$, and when $\mathrm{S}$ made a length estimate, the level of luminance at the test surface was decreased by moving the source of light to the next point on the optical bench, and so on during the first $20 \mathrm{~min}$ of the course of dark adaptation. Although the data of the present study were judgments of the length of a $120-\mathrm{mm}$ arc, the $S$ was occasionally presented with longer or shorter arcs after he had made a judgment of the $120-\mathrm{mm}$ arc at a given level of luminance and before testing at the next lower level.

After approximately $25 \mathrm{~min}$ in the dark, the $\mathrm{S}$ was required to make length estimates first of a moving arc and then of the arc when stationary. These estimates were made at a number of the levels of lighting used during the first $20 \mathrm{~min}$ of dark adaptation. The $\mathrm{S}$ was then given a break of a few minutes outside the experimental room. On his return, he was again light-adapted for a dark-adaptation trial with a light arc line rotating on a dark background. The procedure was the same for the second group of Ss, except that all estimates up to the short break were made with a light arc on a dark surround and then with a dark arc on a light surround. Each $S$ in both groups was allocated to one or the other of the two radial distances, and the arcs were presented to him at this one radial distance throughout the trials. In this second session. all $S$ s thus made comparable length estimates of rotating arc lines under four conditions of stimulus presentation: (a) a light arc during the first 20 min of dark adaptation, (b) a light arc after $25 \mathrm{~min}$ in the dark, (c) a dark arc during the first $20 \mathrm{~min}$, and (d) a dark arc after 25 min of dark adaptation.

\section{Results}

The median estimates of the length of the $120-\mathrm{mm}$ rotating arcs were calculated for each of the six Ss under the four conditions of stimulus presentation of the second session. An analysis of variance gave no significant effect for the order of presentation of light and dark arcs $[F(1,4)=0.32, p>.05]$, but the differential effect of the four conditions was significant $[F(3,12)=6.22, p<.01]$. An inspection of the data indicated that one of the conditions had quite a different effect from the other three. For each of the six Ss, the median estimate of the length of the $120-\mathrm{mm}$ rotating arc taken over all testing luminances was shorter with a light arc during the first 20 min of dark adaptation than with the same arc after $25 \mathrm{~min}$ in the dark or with a dark arc either during the first 20 min or after $25 \mathrm{~min}$ in the dark. The means of the six median estimates in each of the four conditions are 63.5, 139.3, 137.5 , and $135.7 \mathrm{~mm}$, respectively. The component of variation relating to the test of whether length estimates of a light arc during the first $20 \mathrm{~min}$ of the course of dark adaptation differ significantly from estimates obtained under the other three conditions is 246.42 with $\mathrm{F}=21.32, \mathrm{p}<.01$ (Winer, 1962, p. 113). This condition has a significantly different effect on length estimates, as compared with the rest, and the differences among the estimates made in the three other conditions are not significant.

No marked differences were apparent between the length estimates of arcs set at the two radial distances, and these have not been separately analyzed.

At each of the 14 luminance levels presented to the Ss during the first 20 min of dark adaptation, the median of the six length estimates for the dark arc was longer than the median of the estimates of the length of the light arc. Judgments of the light arc tended to be made after a shorter adaptation time than were those of the dark arc, at each level of testing luminance used during the first 20 min of dark adaptation. Thus, for judgments made after the same length of adaptation time, a higher luminance level was needed for detecting the presence of and for judging the length of the dark arc than was needed for the light arc. When length estimates of the two arcs are compared by taking the judgments made by each $\mathrm{S}$ at times (arbitrarily selected at 3-min intervals), the medians of the estimates of the dark arc are significantly longer than those for the light $\operatorname{arc}(t=10.5$, $\mathrm{df}=4, \mathrm{p}<.01$ ). There is no indication from the individual records of any systematic increase in the length estimates of the rotating light arc during the first 20 min of dark adaptation. The median estimates of the 
length of the stationary arcs made by the six Ss when completely dark adapted have a mean of $113.3 \mathrm{~mm}$. Under the condition of a light rotating arc during the first $20 \mathrm{~min}$ of dark adaptation, and under none of the other three conditions of presenting the rotating arcs, the medians of the estimates of length made by each of the six Ss are significantly shorter than the medians of their estimates of the stationary arc $(t=4.43, \mathrm{df}=5$, $\mathrm{p}<.01$ ) and shorter than its actual length of $120 \mathrm{~mm}$. Thus, the apparent contraction effect with a rotating arc was obtained during dark adaptation but only with the condition of a light arc seen during the early course of dark adaptation.

\section{EXPERIMENT II INSTRUCTIONS AND OTHER VARIABLES}

\section{Method}

\section{Subjects}

Twelve undergraduates who had no marked visual defects and no previous experience in the experimental situation attended individually for a session lasting approximately $13 / 2 \mathrm{~h}$.

\section{Apparatus}

The apparatus was essentially as described in Experiment I. The disk was now located $1 \mathrm{~m}$ from the S's chinrest and rotated at either 40 or $70 \mathrm{rpm}$. An arc-shaped slot $(120 \times 4 \mathrm{~mm})$, at a radial distance of $210 \mathrm{~mm}$, could be shortened to $60 \mathrm{~mm}$ or broken by a centrally placed $30-\mathrm{mm}$ gap or by two $30-\mathrm{mm}$ gaps placed symmetrically to yield three $20-\mathrm{mm}$ light sections. These gaps were made by means of black metal plates $30 \mathrm{~mm}$ in width.

\section{Procedure}

Each $\mathrm{S}$ was dark adapted for 25 min before making any judgments and, apart from light at the test surface, remained in the dark throughout his session. Six Ss (randomly selected) were given standard instructions to estimate the length of a rotating light arc in inches or fraction of an inch (Stanley, 1966). The other six Ss were told in addition, "You might see the line as having one or more brighter regions in it. If so, make your estimates of length from the beginning to the end of this brighter region or regions, ignoring any fainter trace or trailing region."

All Ss were required to estimate the lengths of four light ares having the following continuous or gapped lengths: $60 \mathrm{~mm}$, $120 \mathrm{~mm}, 120 \mathrm{~mm}$ with one $30-\mathrm{mm}$ gap, and $120 \mathrm{~mm}$ with two $30-\mathrm{mm}$ gaps. These were presented at rotation speeds of either 40 or $70 \mathrm{rpm}$ and at either of two luminance levels. The higher luminance used was at a photopic level of $2.5 \mathrm{~cd} / \mathrm{m}^{2}$, the lower luminance used was set individually for each $S$. To do this, each $S$ was asked, after the initial period of dark adaptation, to report whether he could see the light from a $60-\mathrm{mm}$ light arc rotating at $70 \mathrm{rpm}$. The light level was reduced in steps until he failed to see the arc. The second level of luminance was set for the $S$ at approximately $0.25 \mathrm{log}$ units above this rough "threshold" for the visibility of the arc with binocular vision.

The estimates of arc length were made monocularly, one eye being occluded. Estimates were made in sets of eight, with one of the four arcs presented at one of the two levels of luminance. Half the estimates in each set were made with the are rotating at $40 \mathrm{rpm}$ and half at $70 \mathrm{rpm}$, the order of these speeds being random. Sets of eight estimates with each of the eight combinations of arc and luminance level were given twice. the order of these sets being random. Apart from a short pause between sets, the presentation of a rotating arc occurred, as in Experiment I, at intervals of approximately $15 \mathrm{sec}$. A warning was given before each estimate had to be made, and the arc line was then exposed for $2 \mathrm{sec}$. The total number of estimates of rotating arcs from each $S$ was 128 . Stationary estimates of apparent length of the $120-\mathrm{mm}$ arc were obtained at the higher luminance level.

\section{Results}

Median estimates were calculated for each $\mathbf{S}$ for each condition. An analysis of variance yielded nonsignificant effects for instructions $[F(1,10)=0.91, p>.05]$, speed $[F(1,10)=3.20, p>.05]$, and gaps $[F(2,20)=2.76$, $\mathrm{p}>.05]$. A significant effect was obtained for light level $[F(1,10)=7.54, p<.05]$, with the lower level $(\bar{X}=$ $172 \mathrm{~mm}$ ) having longer estimates than the higher level $(\overline{\mathrm{X}}=152 \mathrm{~mm})$. The mean difference between the estimates with scotopic and photopic arc luminance for the dark-adapted eye is not great in absolute terms, and the mean estimate for the photopic level is not shorter than the mean estimate for the stationary arc $(\overline{\mathrm{X}}=$ $132 \mathrm{~mm}$ ) or the actual length of the arc.

The 120-mm arc was judged significantly longer than the $60-\mathrm{mm}$ arc $[F(1,10)=65.93, \mathrm{p}<.001]$, and the $120-\mathrm{mm}$ arc with two gaps was also significantly longer than the $60-\mathrm{mm}$ arc $[\mathrm{F}(1,10)=61.99, \mathrm{p}<.001]$.

\section{DISCUSSION}

The results of Experiment I show that the apparent contraction of a rotating light arc and the absence of any contraction of a dark arc, expected under normal viewing conditions, occurs also when the Ss are incompletely dark-adapted. There is no indication that length estimates of the light are tend to increase during the first $20 \mathrm{~min}$ of adaptation, but this may be due to the not unexpected variability of the individual estimates made in these circumstances by untrained Ss. Both experiments demonstrate an absence of apparent contraction when the $\mathrm{S}$ has been dark-adapted for more than $25 \mathrm{~min}$. One possible explanation of this finding is that with the increased sensitivity of the eye, the Ss change the basis of the judgment so that faint traces of the arc, not visible under normal viewing, tend to be included in their length estimates. Such a change in criteria could occur without the increased sensitivity making the display completely homogeneous. The instruction variable of Experiment II was an attempt to get the $\mathrm{S}$ to base his judgment consciously on some possible brighter region or regions, but this did not tend to produce shorter length estimates. Each of the other variables of Experiment II had been previously tried in studies of the contraction effect under normal viewing conditions. When the $\mathrm{S}$ is not dark-adapted, speed of rotation of a light arc is an important variable, but under the conditions of Experiment II. speed of rotation did not affect length estimates. Normally. luminance has only a minimal effect on the degree of contraction. 
After 25 min of dark adaptation, a higher luminance produced shorter estimates than did a lower luminance, but both sets of estimates tended not to be shorter than the actual length of the rotating arc. It has been found that gaps in a light arc do not prevent the contraction that would occur on rotating a continuous arc (Stanley \& Dua, 1969). Experiment II shows that after prolonged dark adaptation, there is no contraction effect with a continuous arc and the presence of gaps in the arc does not change this.

Békésy (1968) reported that Mach bands could not be produced under conditions of dark adaptation, and a similar finding of no apparent contraction of a rotating light arc was anticipated, since the kinds of interactive processes that have been suggested as underlying the two phenomena are analogous. An alternative account of Ansbacher's contraction effect might have been in terms of some more simple pooling of excitation, but such areal summation and pooling might be expected with adequate dark adaptation, and it is then that contraction fails to occur. Experiment II also shows that gaps in a rotating arc do not shorten the length estimates and that a $60-\mathrm{mm}$ continuous arc is seen to be shorter than a $120-\mathrm{mm}$ line with $60 \mathrm{~mm}$ of gapping. Stanley and Dua (1969) noted that under normal viewing it was possible to detect the presence of gaps at rotation speeds that were still fast enough to produce a contraction effect. Ansbacher's phenomenon and Mach bands are both complex phenomena. Interactive, inhibitory processes that give edge effects may accompany and control any pooling of excitations. In the case of the moving arc, failure to establish the leading edge may destroy the possibility of other processes that develop contraction effects. The common absence of the Mach band effect and of the apparent contraction of rotating light arcs when the $S$ is almost completely dark adapted support Stanley's (1967a, 1968) general account of the contraction effect in terms similar to those postulated by Békésy (1967) to account for the Mach band effect.

\section{REFERENCES}

Ansbacher, H. L. Distortion in the perception of real movement. Journal of Experimental Psychology, 1944, 34, 1-23.

Barlow, H. B. Dark-adaptation: A new hypothesis. Vision Research, 1964, 4, 47-58.

Békésy, G. von Sensory inhibition. Princeton, N.J: Princeton University Press, 1967.

Békésy, G. von Mach- and Hering-type lateral inhibition in vision. Vision Research, 1968, 8, 1483-1499.

Graham, C. H. On some aspects of real and apparent visual movement. Journal of the Optical Society of America, 1963, $53,1019-1025$.

Lythgoe, R. J. The mechanism of dark adaptation. A critical resume. British Journal of Ophthalmology, 1940, 24, 41-43.

Marshall, A. J., \& Stanley, G. The apparent length of light and dark arcs seen peripherally in rotary motion. Australian Journal of Psychology, 1964, 16, 120-128.

Stanley, G. Apparent shrinkage of a rotating arc as a function of luminance relations between figure and surround. Acta Psychologica, 1966, 25, 357-364.

Stanley, G. Light summation and the perceived length of moving lines. Acta Psychologica, 1967a, 26, 260-264.

Stanley, G. Apparent brightness of a rotating arc-line as a function of speed of rotation. Acta Psychologica, 1967b, 26, 17-21.

Stanley, G. Exposure-time and the apparent length of an illuminated rotating arc-line. Perception \& Psychophysics, $1968,4,71-72$.

Stanley, G., \& Dua, J. K. The effect of dark gaps on the apparent length of a moving light line. Acta Psychologica, 1969, 31 , 189-193.

Stanley, G., \& Jackson, R. The effect of leading contour on the relative lengths of moving light and dark arcs. Journal of Psychology, 1969, 71, 83-88.

Winer, B. J. Stritistical principles in experimental design. New York: McGraw-Hill, 1962.

(Received for publication March 5, 1973; revision received May $16,1973$. ) 